\title{
Time-Related Heart Vegetative Balance Characteristics in Healthy Subjects
}

\author{
M Matveev ${ }^{1}$, R Prokopova $^{2}, \mathrm{Ch} \mathrm{Nachev}^{2}$ \\ ${ }^{1}$ Centre of Biomedical Engineering Bulgarian Academy of Sciences, Sofia, Bulgaria \\ ${ }^{2}$ Department of Internal Medicine St Anne University Hospital, Sofia, Bulgaria
}

\begin{abstract}
Abs tract
Changes of the heart autonomic balance between morning $(8$ to $9 \mathrm{~h})$ and afternoon $(14-15 \mathrm{~h})$ measurements were studied in 22 healthy subjects. The selection of these two daytime periods was substantiated by the established higher risk of cardiovascular incidents in the morning and the relative balance of the vegetative nervous system in the afternoon hours. The changes were analyzed by RRvariability indices from ECG recordings in resting state and with vegetative nervous system stimulation by handgrip test and Valsalva maneuver. The authors introduced an indicator for time-related autonomic balance changes. The indicator evaluates the power of each $R R$-variability index to respond to changes in the autonomic control, in comparisons between resting state and stimulation data in the morning and afternoon measurements.
\end{abstract}

\section{Introduction}

The disturbed balance of the vegetative nervous system (VNS) attends or leads to various cardiovascular diseases.

Heart autonomic control can be studied by timedomain and frequency-domain indices of RR variability (RRV) [1-2].

VNS balance changes round the clock [3-4]. It is natural to connect circadian autonomic balance changes with increased vascular incidents in specific time intervals. The period between $6 \mathrm{~h}$ and $10 \mathrm{~h}$ has been shown as especially hazardous [3], [5-7].

The above-cited studies confirm the existence of time-related changes in the HAB. Due to discrepancy in the tendencies of these changes derived from Holter ECG studies, we undertook studying HAB changes in healthy subjects in two morning and afternoon hour intervals. This choice was motivated by the proven high morning risk of cardiovascular incidents and the relatively stable HAB balance in the afternoon hours.

\section{Material and mathematical- statistical approach}

The study included 22 healthy subjects, 11 men and 11 women, mean age 47.3 years (range $21-76$ ), with no anamnesis data of cardiovascular and neurological disease, organ insufficiency and diabetes. Their previous ECG and blood pressure Holter recordings did not reveal arterial hypertension and ishaemic heart disease. In order to detect small changes in $\mathrm{HAB}$, we used RR-interval recordings in resting state and in VNS stimulation with handgrip test and Valsalva maneuver. RRV indices for each individual were measured from 512 RR-intervals in resting state, $128 \mathrm{RR}$-intervals with handgrip test and 64-intervals with Valsalva maneuver, obtained in the morning (8-9 h) and afternoon (14-15 h). Before electrocardiogram recording the subjects were in lying position for $30 \mathrm{~min}$, with ambient air temperature $22-24{ }^{\circ} \mathrm{C}$. The ECG signals were sampled with $4000 \mathrm{~Hz}$ and with $2.5 \mu \mathrm{V}$ digital resolution (dynamic range: $+/-10 \mathrm{mV}$ AC). The tests were performed in the lying position in $20 \mathrm{~min}$ intervals. Standardized indices for RRV were used [1].

To evaluate morning and afternoon differences in $\mathrm{HAB}$, we used an adequate statistical criterion - the sign test, e.g. [8].

In order to assess the power of each RRV index to respond to time-related changes in the $\mathrm{HAB}$, we introduced a specific 'autonomic balance' indicator (TRABI). Let two measurements are made in two different moments of time, in order to detect the influence of a factor in two tests. In each measurement $\mathrm{i}(\mathrm{i}=1,2)$ we obtain values of an index $\mathrm{V}$ for $\mathrm{N}$ objects in two tests. For each measurement, comparing the $\mathrm{N}$ parallel pairs of values of the index $\mathrm{V}$, we have $\mathrm{k}_{\mathrm{i}}(+)$ 
positive, $\mathrm{k}_{\mathrm{i}}(-)$ negative and $\mathrm{k}_{\mathrm{i}}(0)$ zero differences. Then the following limitation is valid:

(1) $\quad \mathrm{k}_{\mathrm{i}}(+)+\mathrm{k}_{\mathrm{i}}(-)+\mathrm{k}_{\mathrm{i}}(0)=\mathrm{N}(\mathrm{i}=1,2)$

Let us consider the quantity

$$
\begin{aligned}
\mathbf{I} & =\left|\left[\mathrm{k}_{1}(+)-\mathrm{k}_{1}(-)\right]-\left[\mathrm{k}_{2}(+)-\mathrm{k}_{2}(-)\right]\right| / 2 \mathrm{~N}= \\
& =\left|\mathrm{dk}_{1}-\mathrm{dk}_{2}\right| / 2 \mathrm{~N},
\end{aligned}
$$

where, taking into account (1), the zero differences $\mathrm{k}_{\mathrm{i}}(0)$ are indirectly included through $\mathrm{N}$. Obviously, the indicator introduced by (2) measures the power of the index to respond to changes in comparing results from two tests in two measurements, performed in different moments of time (for example, morning and afternoon).

Equations (1) and (2) define a range of values of $[0,1]$ for I. In order to illustrate the conditions for obtaining the values of $\mathbf{I}$, we assume for convenience (without influencing the essence of the results), that there are no zero differences in the two measurements. Hence,

(3) $\quad \mathrm{k}_{\mathrm{i}}(0)=0$ and $\mathrm{N}=\mathrm{k}_{\mathrm{i}}(+)+\mathrm{k}_{\mathrm{i}}(-)$.

(The presence of zero differences reduces the values of the indicator (2), depending on their number).

Obviously, the indicator is zero if $\mathrm{dk}_{1}=\mathrm{dk}_{2}$. Considering (3), this is possible only if both equalities $\mathrm{k}_{1}(+)=\mathrm{k}_{2}(+)$ and $\mathrm{k}_{1}(-)=\mathrm{k}_{2}(-)$ are satisfied. In other words, the indicator value is zero (no time-related changes ) only in case in the two measurements there is no change in the number of positive and negative differences of the index $\mathrm{V}$ values obtained from two tests.

The maximum value $\mathrm{I}=1$ is defined from $\mid \mathrm{dk}_{1}-$ $\mathrm{dk}_{2} \mathrm{l}=2 \mathrm{~N}$ and considering (3), it is necessary that $\mathrm{dk}_{1}=-$ $\mathrm{dk}_{2}$, or satisfaction of one of the two conditions $\left\{\mathrm{k}_{1}(+)=\mathrm{N}, \mathrm{k}_{1}(-)=0 ; \mathrm{k}_{2}(+)=0, \mathrm{k}_{2}(-)=\mathrm{N}\right\}$, or $\left\{\mathrm{k}_{1}(+)=0, \mathrm{k}_{1}(-\right.$ )$\left.=\mathrm{N} ; \mathrm{k}_{2}(-)=\mathrm{N}, \mathrm{k}_{2}(-)=0\right\}$. There is a case of full change in the number of positive and negative differences of the index $\mathrm{V}$ values from the two measurements and the two tests.

\section{Results}

\subsection{Differences between morning and afternoon values of $R R V$ indices in resting state, handgrip test and Valsalva maneuver}

The results show no significant changes between morning and in the afternoon RRV indices measured with all three tests.

\subsection{Differences between values of RRV indices in resting state and stimulation tests}

Morning measurements. In comparison of resting state with handgrip test, significant changes $(p<0.05)$ can be seen in two time-domain indices (RRSD and RRMD) and in the three frequency-domain indices (VLF, LF and HF). The handgrip test values of LF and HF decreased with respect to the resting state values, with a high significance difference $(\mathrm{p}<0.0001)$.

There were different results in comparison of resting state with Valsalva maneuver. The indices constellation with significant value changes did not include RRA and MED only. The frequency-domain indices LF and HF are again of lesser values, compared to resting state. For HF, changes were found in all subjects. (The Valsalva test did not include VLF, due to the short ECG record duration).

The LF/HF index did not belong to the constellations, for the two types of comparison between resting state and handgrip test and between resting state and Valsalva maneuver.

Afternoon measurements. The time-domain indices RRA, DRRA, DRRSD, DRRMD, PNN50 and RMS showed significant differences of handgrip test values, compared to resting state. It is important to note that except for RRA, these indices are from the dRRtachogram. Three of the frequency-domain indices (VLF, LF and HF) also registered significant differences.

Comparing the values in resting state with those from Valsalva maneuver, only two time-domain indices showed significant difference of marginal level, namely DRRSD and DRRMD. The frequency-domain indices LF and HF showed significant changes, but of lower level in comparison with handgrip test. LF/HF did not show significant difference between resting state and handgrip test and between resting state and Valsalva maneuver.

The results from the two previous sections showed: i) no significant differences between morning and afternoon values of RRV indices for all three types of measurements; ii) statistically significant differences between resting state and stimulation values, but in different indices constellations for the morning and afternoon measurements. Therefore, it was justified to look for relative changes in time-related HAB indices. First the indices values in resting state and with stimulation in morning measurements are compared. Next the same comparison is done for afternoon measurements. The power of the RRV indices to detect the time-related differences between these two comparisons is evaluared by indicator for time-related autonomic balance changes (TRABI). The evaluation results for th? morning and afternoon measurements in resting state and with handgrip test are shown in Table 1. The results for resting state and Valsalva maneuver are shown in Table 2. These two tables include the number of negative $\mathrm{k}_{\mathrm{i}}(-)$ and positive $\mathrm{k}_{\mathrm{i}}(+)$ differences in resting state and stimulation tests in the morning 
$(i=1)$ and afternoon $(i=2)$. In addition, the number of zero differences $\mathrm{k}_{\mathrm{i}}(0)$ is shown, as the latter are indirectly included in the computation of the indicator value.

\section{Discussion}

The TRABI values corresponding to morning and afternoon differences in handgrip stimulation versus resting state divide the RRV indices in two groups with respect to their power to reflect the changes: i) low and insignificant (RRA, RRSD, RRMD, MED, DRRSD, DRRMD, PNN50, RMS, VLF and LF), and ii) moderate (DRRA and LF/HF). For the indices of the first group lower values prevail in morning and afternoon measurements. The relations of positive and negative changes of these indices between morning and afternoon values can be explained by the already shown relative stability of autonomic balance in healthy subjects. A certain difference can be observed for PNN50 and RMS: in the afternoon measurements the number of positive resting state-to-handgrip test differences increases. We explain finding with the power of dRR-tachogram indices to respond to the relatively lower sympathetic level in the afternoon they react to increased adjacent RR interval differences. The assessments for HF and LF by TRABI confirm the assumption for the limited power of these indices to respond to time-related autonomic balance changes.

The higher indicator values for the two indices of moderate sensitivity DRRA and LF/HF are due to different tendencies. For DRRA, the ratio of positive and negative morning and afternoon differences shows more expressed changes in comparison with the indices of the low power group: from 13 positive and 9 negative in the morning, to 17 positive and 4 negative in the afternoon (Table 1). This tendency is better expressed than by PNN50 and RMS. For LF/HF, the tendency is changing - the ratio of positive and negative differences in the morning and afternoon are opposite. For handgrip test, the morning value is lower in 14 subjects and higher in 8 ones, while the afternoon values are lower in 9 subjects and higher in 13. The transition to prevailingly higher values (handgrip test versus resting state) in the afternoon is due to higher LF values in stimulation. It was already commented, that these higher values are possible because of the lower afternoon sympathetic tone, yielding additional stimulation.

The RR-variability indices are also divided in two groups by the autonomic balance indicator, with respect to their power to respond to changes in the morning and afternoon autonomic balance, due to Valsalva maneuver stimulation, with respect to resting state balance. RRSD, RRMD, MED, DRRSD, DRRMD,
PNN50, LF, HF and LF/HF have values below 0.20 (insignificant power). The low indicator values are due to the prevailingly higher values of the indices in stimulation with respect to resting state, both for morning and afternoon measurements. In contrast to the data for handgrip test, no close relationship can be found between positive and negative differences in morning and afternoon results. There is a tendency toward reduced number of negative differences in the afternoon. This is especially expressed for RRSD, RRMD, and in a lesser extent for DRRSD and PNN50 (Table 2). This tendency might be due to the limited power of Valsalva maneuver to stimulate the relatively more excited parasympathetic system in the afternoon. The low indicator values for LF and HF is due to the virtually equal ratio of morning and afternoon positive and negative differences.

The group of moderate power to represent the changes includes RRA, DRRA and RMS. The reason for DRRA and RMS is a more expressed change in the ratio of morning and afternoon positive and negative differences, but with still prevailing negative differences. For RRA this tendency has already changed the ratio of positive and negative differences: from 14 negative and 7 positive in the morning, to 6 negative and 16 positive in the afternoon (Table 2). This yields the highest indicator value for this index (0.386). The reason for changes in the mean RRinterval, i.e. the mean cardiac frequency, can be interpreted by the need for mastering the lower afternoon sympathetic tone, in order to restore the autonomic balance during and after Valsalva maneuver.

\section{Conclusions}

The heart autonomic balance in healthy subjects is characterized by relative stability in time. In spite of this relative stability, RR-variability indices can reveal time-related autonomous balance changes: relatively lowerparasympathetic tone and hypersympatheticotony were present in the risky morning hours.

The power of RR-variability indices to respond to time-related changes in the autonomic balance due to stimulation is different. The frequency-domain indices have low sensitivity for changes and do not take part in the composition of a time-related heart autonomic balance profile in healthy subjects. The time-domain indices have considerably higher power to react to time-related changes in the balance in stimulation of of the vegetative nervous system components.

The proposed indicator for assessment of timerelated changes is an adequate quantitative measure for the individual power of the RR-variability indices to respond to changes in the heart autonomic balance. Specific time-related changes in subjects with 
cardiovascular disease in initial and/or advanced stage

profile of changes in healthy subjects.

can be defined, by comparison with the corresponding

Table 1. Values of TRABI for the RRV indices in comparison of morning and afternoon measurements in resting state and with handgrip test

\begin{tabular}{|c|c|c|c|c|c|c|c|c|c|}
\hline \multirow{2}{*}{ Indices } & \multicolumn{4}{|c|}{ Morning measurements } & \multicolumn{4}{|c|}{ Afternoon measurements } & \multirow{2}{*}{ TRABI } \\
\hline & $N$ & $k_{l}(-)$ & $k_{1}(+)$ & $k_{l}(0)$ & $N$ & $k_{2}(-)$ & $k_{2}(+)$ & $k_{2}(0)$ & \\
\hline RRA & 22 & 7 & 15 & 0 & 22 & 5 & 17 & 0 & 0.091 \\
\hline RRSD & 20 & 4 & 16 & 2 & 22 & 6 & 16 & 0 & 0.045 \\
\hline RRMD & 21 & 5 & 16 & 1 & 20 & 6 & 14 & 2 & 0.068 \\
\hline MED & 22 & 7 & 15 & 0 & 22 & 6 & 16 & 0 & 0.045 \\
\hline DRRA & 22 & 9 & 13 & 0 & 21 & 4 & 17 & 1 & 0.205 \\
\hline DRRSD & 19 & 6 & 13 & 3 & 21 & 5 & 16 & 1 & 0.091 \\
\hline DRRMD & 19 & 6 & 13 & 3 & 22 & 5 & 17 & 0 & 0.114 \\
\hline PNN50 & 16 & 5 & 11 & 6 & 19 & 3 & 16 & 3 & 0.159 \\
\hline RMS & 22 & 8 & 14 & 0 & 21 & 4 & 17 & 1 & 0.159 \\
\hline VLF & 22 & 1 & 21 & 0 & 22 & 3 & 19 & 0 & 0.091 \\
\hline $\mathrm{LF}$ & 22 & 0 & 22 & 0 & 22 & 1 & 21 & 0 & 0.045 \\
\hline $\mathrm{HF}$ & 22 & 0 & 22 & 0 & 22 & 0 & 22 & 0 & 0.000 \\
\hline $\mathrm{LF} / \mathrm{HF}$ & 22 & 8 & 14 & 0 & 22 & 13 & 9 & 0 & 0.227 \\
\hline
\end{tabular}

Table 2. Values of TRABI for the RRV indices in comparison of morning and afternoon measurements in resting state and with Valsalva maneuver

\begin{tabular}{|c|c|c|c|c|c|c|c|c|c|}
\hline \multirow{2}{*}{ Indices } & \multicolumn{4}{|c|}{ Morning measurements } & \multicolumn{4}{|c|}{ Afternoon measurements } & \multirow{2}{*}{ TRABI } \\
\hline & $N$ & $k_{l}(-)$ & $k_{l}(+)$ & $k_{1}(0)$ & $N$ & $k_{2}(-)$ & $k_{2}(+)$ & $k_{2}(0)$ & \\
\hline RRA & 21 & 14 & 7 & 1 & 22 & 6 & 16 & 0 & 0.386 \\
\hline RRSD & 22 & 19 & 3 & 0 & 22 & 15 & 7 & 0 & 0.182 \\
\hline RRMD & 22 & 19 & 3 & 0 & 22 & 15 & 7 & 0 & 0.182 \\
\hline MED & 22 & 14 & 8 & 0 & 22 & 11 & 11 & 0 & 0.136 \\
\hline DRRA & 21 & 17 & 4 & 1 & 22 & 13 & 9 & 0 & 0.205 \\
\hline DRRSD & 22 & 20 & 2 & 0 & 21 & 16 & 5 & 1 & 0.159 \\
\hline DRRMD & 22 & 18 & 4 & 0 & 20 & 15 & 5 & 2 & 0.091 \\
\hline PNN50 & 21 & 17 & 4 & 1 & 20 & 13 & 7 & 2 & 0.159 \\
\hline RMS & 22 & 18 & 4 & 0 & 22 & 13 & 9 & 0 & 0.227 \\
\hline $\mathrm{LF}$ & 22 & 3 & 19 & 0 & 22 & 4 & 18 & 0 & 0.045 \\
\hline $\mathrm{HF}$ & 22 & 0 & 22 & 0 & 22 & 2 & 20 & 0 & 0.091 \\
\hline LF/HF & 22 & 11 & 11 & 0 & 22 & 14 & 8 & 0 & 0.136 \\
\hline
\end{tabular}

\section{References}

[1] Heart rate variability: standards of Measurements, physiological interpretation and clinical Use. Task Forse of the European Society of Cardiology and the North American Society of Pacing and Electrophysiology. Circulation 1996;93: 1043-65.

[2] Aubert AE, Beckers F, Verheyden B. Heart rate Variability. Methods and Applications. Proc. Of the $19^{\text {th }}$ European Congress of the Intern. Soc. of Non-Inv. Card., Istanbul, May, 2003:144-146.

[3] Arshed A. Circadian rythms in cardiovascular disease. Am Heart J 1990;120:726-33.

[4] Molgaard H, Sorensen KE, Bjerregaard P. Circadian variation and influence of risk factors on heart rate variability in healthy subjects. Am J Cardiol 1991;68:777-84.
[5] Cari JP. Circadian Variations in Myocardial Ishemia. JAMA 1991;265:386-390.

[6] Heikki V, Huikuri HV, Linnaluoto M. Circadian Rhythm of Heart Rate Variabilty in Survivors of Cardiac Arrest. Am J Cardiol 1992;70:610-615.

[7] Casetta I, Granieri E, Fallica E, La Cecilia O et al. Patient demographic and clinical features and circadian variation in onset of ischemic stroke. Arch Neurol 2002; 59:48-54.

[8] Conover WJ. Practical Nonparametric Statistics. New York: John Wiley \& Sons, 2nd ed.:1980.

Address for correspondens

Mikhail Matveev

CLBME, Acad. G.Bonchev Str., B1.105, 1113 Sofia, Bulgaria

E-mail address: mgm@clbme.bas.bg 